Boletín de la Sociedad Geológica Mexicana

VOLUMEN 66, NÚM. 1, 2014, P. 223-224

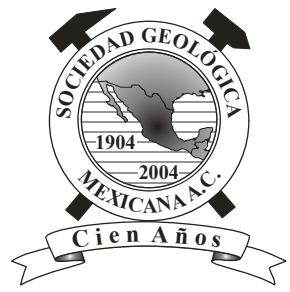

\title{
Revisión
}

\section{The Soils of Mexico}

\author{
Miguel A. Segura-Castruita, ${ }^{1, *}$ \\ ${ }^{1}$ División de Estudios de Posgrado en Investigación, Instituo Tecnológico de Torreón, Carretera Torreón-San Pedro km 7.5, Torreón, \\ Coahuila, C.P. 27170, México. \\ *dmilys5@hotmail.com
}

El suelo como cuerpo tridimensional, viviente y dinámico, es parte integral del ecosistema terrestre de nuestro planeta, componente importante en el suministro de bienes y servicios para el ambiente global y para el hombre. El recurso suelo varía gradualmente en el espacio geográfico y a través del tiempo, forma complejos patrones por la interrelación de múltiples factores ambientales y funciones naturales forzadas. Por ello, parte de las investigaciones que desarrollan los científicos del suelo, a diferentes escalas de observación, se han realizado con la disciplina integradora conocida como Pedología. Esta disciplina se enfoca en el modo de formación de los suelos, composición, factores que influyen en ellos y su distribución geográfica; de tal manera que, en México y en el mundo se ha generado conocimiento de este recurso, como se muestra en el libro The Soils of Mexico (Krasilnikov et al., 2013)

The Soils of Mexico fue escrito por un equipo de seis investigadores con amplia trayectoria y reconocido prestigio académicocientífico a nivel internacional en el área de la pedología, dentro de la Ciencia del Suelo. Esta obra forma parte de la colección de libros World Soils Book Series, editada por el Profesor Alfred E. Hartemink, bajo el auspicio de la International Union of Soil Science (IUSS).

El libro integra información científica acerca del suelo con un enfoque pedológico, donde se hace referencia a artículos científicos, de divulgación, memorias de congresos y libros, así como documentos históricos y de diferentes dependencias federales de México, con el fin de mostrar claramente, a través de un análisis, cuál es el estado del arte del conocimiento sobre los suelos de México. Cuenta con una introducción general y siete capítulos escritos en idioma inglés, con una redacción ligera y entendible, que hacen amena e interesante la lectura. Enseguida se menciona de manera general el contenido de este epítome.

La Introducción da una explicación del por qué el libro fue escrito y se plantean de manera brillante las conclusiones de esta obra, donde se establecen las diferentes relaciones entre los factores formadores del suelo (material parental, relieve, clima, biota y tiempo) y los procesos de formación, para dar como resultado la gran gama de suelos que se encuentran en las diferentes regiones de México. Asimismo, se menciona la aplicación de la geoestadística a partir de las propiedades intrínsecas del suelo, para generar mapas predictivos del mismo. Continúa con la presencia de paleosuelos en el país como resultado de los cambios en el ambiente y finalmente, se reconoce la complejidad de la distribución de los suelos en el territorio mexicano y la necesidad de continuar estudiándolos.

El segundo capítulo, Soil Reserach and Mapping History (Investigación de Suelos e Historia de la Cartografía de Suelos), está dividido en cuatro apartados, donde se presenta una relatoría sintetizada del conocimiento tradicional que data de épocas prehispánicas y que en nuestros días continúa vigente, haciendo hincapié en el papel que México ha tenido en la generación de conocimiento y su utilidad en los estudios de suelo. Además de la reseña histórica de la Ciencia del Suelo en el país, resalta la participación de investigadores mexicanos y extranjeros, así como de instituciones de educación e investigación y del gobierno federal para, en primer lugar, traer el conocimiento del suelo a México y posteriormente desarrollar esta ciencia. Este capítulo concluye con una narración sucinta de la evolución de los mapas edáficos en México, con ilustraciones que demuestran el perfeccionamiento de la cartografía, desde los códices elaborados en épocas prehispánicas, hasta la actualidad donde se utilizan las tecnologías de la información para elaborar diferentes mapas temáticos.

El capítulo número tres, Factors of Soil Formation (Factores de Formación de Suelo), está dividido en cuatro apartados. Inicia con una descripción general de la geografía del territorio nacional. Continúa con la descripción de la orografía, geología, geomorfología, clima, hidrología, flora y fauna de México; se establecen diferentes relaciones entre ellos, haciendo un análisis para explicar de manera general, la distribución y diversidad de rocas, relieves, climas y seres vivos (flora y fauna) que existen en estas latitudes. 
Este análisis sentó las bases para la comprensión de la existencia de los diferentes grupos o clases de suelo en México.

El cuarto capítulo, Major Soil Types and Their Classification (Principales Tipos de Suelo y su Clasificación), está integrado por dieciséis apartados, en los que se indican los sistemas empleados en el país para clasificar los tipos de suelo, en función de su origen y evolución, para finalizar con la conclusión del capítulo. Es importante resaltar que en cada uno de los apartados se mencionan las características generales de los suelos en las diferentes agrupaciones (provisionales) que se presentan, considerando datos que se encuentran en la literatura, así como fotografías de perfiles representativos y en algunos casos de la microestructura o características pedogenéticas que éstos tienen.

La Regionalización Geográfica del Territorio Mexicano (Geographical Regionalization of the Mexican Territory) es el quinto capítulo; en esta sección México es dividido en quince regiones y se explica el por qué de la regionalización y los fines de la misma. Toman como base las Provincias Fisiográficas en las que está dividido México, que para este libro se consideraron como regiones pedográficas. En cada una de las regiones aparece una descripción de la topografía, geología, hidrología, clima, vegetación, suelos y el uso, manejo y degradación del recurso edáfico, con ilustraciones y mapas.

El capítulo sexto, Soil Degradation (Degradación de Suelos), está dividido en seis partes; contiene una explicación de la evolución histórica de la degradación del suelo en nuestro país, relacionando los factores clima, relieve, vegetación y la influencia humana en la aceleración o desaceleración de este proceso. Igualmente, se presenta información relacionada con la erosión y desertificación, donde se hace un análisis de las diferentes metodologías que se han utilizado para estudiarlas, así como del estado que guarda el territorio nacional a causa de estos problemas. Finalmente, se mencionan algunas áreas de oportunidad para llevar a cabo investigaciones relacionadas con la degradación del suelo.

El séptimo capítulo, Soil and Human Throughout the History (Suelo y Humano a lo Largo de la Historia), trata de la importancia que ha tenido el suelo en la producción de alimentos, tanto para nuestros antepasados como para el mexicano actual. También, da un recorrido por las diferentes culturas mesoamericanas que existieron en México, y se establece la relación que tuvo la distribución de sus poblaciones con los suelos existentes en los lugares de asentamiento; así como la implementación de diferentes técnicas (de conservación o producción agrícola) desarrolladas por los pobladores, para obtener alimentos en esos lugares, y que aún en nuestros días continúan vigentes. Este capítulo concluye con una reflexión acerca de la importancia de los suelos y su manejo; además, de la necesidad de un mejor entendimiento de las técnicas tradicionales a fin de realizar una agricultura sustentable.

El capítulo final, Paleosols of Mexico: Origin, Paleoecological Significance, Role in the Actual Soil Mantle (Paleosuelos de México: Origen, Significado Paleoecológico, Papel en la Capa de Suelo Actual), reúne un cúmulo de información relacionada con la presencia de paleosuelos en el territorio nacional. La información está dividida en tres partes: la primera es una breve introducción a la paleopedología. La segunda ofrece información de paleosuelos enterrados, donde se establece una relación con el tiempo transcurrido, su contexto geológico y el significado paleoecológico. En la tercera se menciona la presencia de paleosuelos y su correspondencia con el paisaje suelo actual, a fin de considerarlos como una memoria de información y su conexión con las funciones medio ambientales del presente.

Sin duda alguna, el libro The Soils of Mexico es una obra importante para el acervo bibliográfico de los científicos del suelo de México, pues a partir de referencias históricas y científicas de los suelos de este territorio se presenta una interpretación acerca del origen, forma y distribución del recurso edáfico en este país, con un enfoque netamente pedológico. Además, establece el estado del arte de la Ciencia del Suelo en su área pedológica, a fin de continuar y profundizar con la generación de conocimientos acerca de los suelos, lo cual lo hace único en su tipo.

\section{Referencias}

Krasilnikov, P., Gutiérrez-Castorena, M.C., Ahrens, R.J., Cruz-Gaistardo, C.O., Sedov, S., Solleiro-Rebolledo, E., 2013, The Soil of Mexico: Netherlands, Springer $187 \mathrm{p}$.

Manuscrito recibido: Abril 3, 2013.

Manuscrito corregido recibido: Mayo 13, 2013.

Manuscrito aceptado: Mayo 14, 2013. 